\title{
PYRENE EMISSION FROM MONOLAYERS "CLICKED" ONTO QUARTZ
}

\author{
HONGYAN SONG, SERGEY GORELIK and JONATHAN HOBLEY* \\ Institute of Materials Research and Engineering (IMRE) \\ Agency for Science Technology and Research $\left(A{ }^{*} S T A R\right)$ \\ 3 Research Link, 117602, Singapore \\ *hobleyj@imre.a-star.edu.sg \\ JIEXUN LI, AÏCHA BOUDHAR and MARTIN J. LEAR \\ Department of Chemistry \\ National University of Singapore (NUS) \\ 3 Science Drive 3, 117543, Singapore \\ †martin.lear@nus.edu.sg
}

Received 8 August 2012

Accepted 8 October 2012

Published 14 December 2012

\begin{abstract}
A series of quartz surfaces were modified with a series of crosslinkers and functional groups in order to obtain an azide-terminated monolayer, which was then used to immobilize pyrene onto the surface via alkyne-azide "click" chemistry. During the course of the immobilization, different ratios of tert-butyl diphenyl chlorosilane were used to control the distribution and hence the photophysical properties of the pyrene on the surface. The preparative surface reactions and photophysical properties were investigated with contact angle, X-ray photoelectron spectroscopy, UV-visible absorption and emission spectroscopy. High surface coverage was achieved of just under 1 molecule per $\mathrm{nm}^{2}$. At this coverage all emission from the pyrene was in the form of excimer emission. Excimer emission dominated at all surface coverages greater than 0.45 molecules per $\mathrm{nm}^{2}$. Below this coverage the monomer emission could also be observed. The conclusions that can be drawn are important for understanding the interactions of neighboring molecules in molecular monolayers. Our results suggest that at high surface coverage a substantial number of the pyrene molecules are already close enough to their neighbors that pairs of them can be directly excited to form excimer with no requirement for diffusion. This can be stated because the long wavelength end of the pyrene absorption and excitation spectra show a broad tail that is assigned to a charge transfer band resulting from an electron being directly transferred from a ground state pyrene to a neighboring pyrene molecule. Furthermore, absorption spectra shifts also indicate that the pyrene molecules undergo some interactions on the surface when they are closely packed.
\end{abstract}

Keywords: Pyrene; excimer; chemisorption; click chemistry; fluorescence probes; self-assembly. 


\section{Introduction}

For a rather simple looking molecule, pyrene has a complex photochemistry, particularly in its formation of excimers and exciplexes., ${ }^{1,2}$ When two ground-state pyrene molecules are brought together in solution, they do not experience strong mutual attraction. However, if one of the molecules is in its first electronically excited state, the two molecules are able to form an excimer. This excimer is a new electronically excited entity with its own electronic and geometric structure. The excimer can undergo fluorescence, however, the lack of a bound ground state of the excimer usually means that any emission should lead to the dissociation of the complex. ${ }^{1}$ This means that the absorption spectrum of a ground state excimer cannot usually be obtained as there is no ground state dimer.

Pyrene's photochemistry has been extensively studied in solution, in micelles, in bilayers, in cells and on a variety of substrates including photocurrent studies on gold surfaces ${ }^{2}$ and intermolecular communication studies on silica surfaces. ${ }^{1-6}$ Pyrene is typically chosen as a sensor fluorophore because of its well-known Ham effect ${ }^{9}$ where the relative intensity of the vibrational bands is sensitive to solvent polarity. Furthermore the complexity of the emission results in various environmental sensitivities leading to spectral shifts. With increasing concern over the environment and ecosystem, pyrene films have been developed as efficient sensors for explosives and dicarboxylic acids, ${ }^{8-11}$ although these have not yet been commercialized. The current work aims to develop an efficient method of coupling pyrene to a surface and to quantify the surface coverage of pyrene. Further, we aim to assess at what surface coverages the excimer and monomer emissions dominate, since it is these emissions that are crucial for any sensor application.

In our study, we used click chemistry as a general method for efficiently creating photoactive monolayers on quartz substrates. "Click chemistry", first coined by Barry Sharpless, ${ }^{12-15}$ is a reaction that is highly chemoselective, it typically is almost quantitative in conversion of starting material to product, it only requires mild reaction conditions and it is orthogonal with biological function and modular. ${ }^{16}$ As a result, it has been widely applied in many fields, including, biochemistry, ${ }^{17}$ macromolecular chemistry $^{18}$ and surface chemistry. ${ }^{19}$
In the area of self-assembled monolayer (SAM), there have been a few reports of click chemistry on gold, ${ }^{20}$ silica $^{21}$ and glass ${ }^{22}$ surfaces. In this paper, we report our work on functionalizing the surface of quartz with pyrene using click chemistry and the relationship between surface coverage and pyrene monomer/excimer formation upon photoexcitation. Further, we show evidence for charge-transfer (CT) absorption at high concentrations of pyrene at the surface produced using this synthetic approach.

\section{Experimental}

All chemicals and solvents were purchased from Sigma-Aldrich. The synthesized pyrene derivative was verified by ${ }^{1} \mathrm{H}$ NMR (Bruker ACF400) and ESIMS (Finnigan/Mat TSQ7000 spectrometer). The extent of the reaction was monitored by thin layer chromatography (TLC) using Merck 60 F254 precoated silica gel plates with fluorescent indicator UV254. The substrates used for modification in this study were quartz $(21 \mathrm{~mm} \times 21 \mathrm{~mm})$.

\subsection{Preparation of aminated surfaces at various coverages}

The quartz slides were chemically cleaned by rinsing several times with deionized water and ethanol, and then dried under a nitrogen stream. Next, they were physically cleaned by baking them in a Nabatherm C furnace at $500^{\circ} \mathrm{C}$ for $5 \mathrm{~h}$. Note from Table 1 that the water contact angel of these cleaned substrate surfaces, obtained using a RaméHart contact angel goniometer, was around $3^{\circ}$. A solution of aminopropyltriethoxysilane (APTES) was prepared by dissolving $0.25 \% \mathrm{v} / \mathrm{v}$ of APTES in dry Toluene. A solution of $0.85 \% \mathrm{v} / \mathrm{v}$ tert-butyldiphenylchlorosilane (TBDPSCl) in toluene was prepared and an appropriate molar ratio of APTES was then added to the solution (Table 2). The clean quartz slides were then incubated in the solution for $30 \mathrm{~min}$ at room temperature. The slides were then washed thoroughly with toluene, ethanol and acetone. The aminated slides were then dried by baking them in an oven for $1 \mathrm{~h}$ at $140^{\circ} \mathrm{C}$.

\subsection{Verification of click chemistry at the quartz surface}

A solution of 5-bromopentanoic acid $(1 \mathrm{mM})$ and 1-ethyl-3-(3-(dimethylamino)propyl)-carboiimide 
(EDC) $(10 \mathrm{mM})$ in ethanol was prepared. The aminated slides were incubated in the solution at room temperature with gentle shaking for $3 \mathrm{~h}$. The slides were removed and washed thoroughly with ethanol and acetone. The brominated slides were immersed in a saturated solution of $\mathrm{NaN}_{3}$ in $\mathrm{DMF}$ at $85^{\circ} \mathrm{C}$ for $48 \mathrm{~h}$ and washed thoroughly with water and acetone to yield the azid-functionalized quartz slides.

Using a $\mathrm{CuBr}$ catalyst ${ }^{23}$ from commercial sources the azido quartz slides were incubated in a solution of alkyne and $\mathrm{CuBr}$ overnight with gentle shaking which yielded the triazole product on the quartz surface (Scheme 1). Each step and the extent of the surface modification and click chemistry as shown in scheme 1 were checked using XPS (Fig. 1). Since propargyl bromide was selected as the alkyne source its incorporation via click chemistry could be checked via XPS using its characteristic C-Br peak. Using XPS, we were able to determine that the
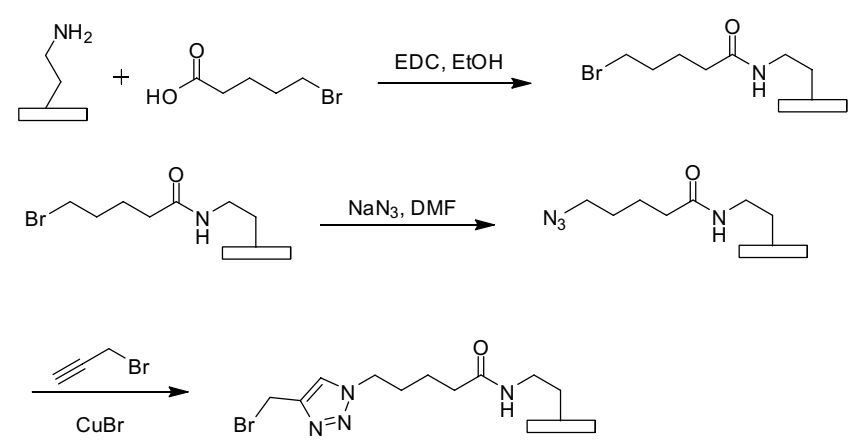

Scheme 1. Surface aziridation route.

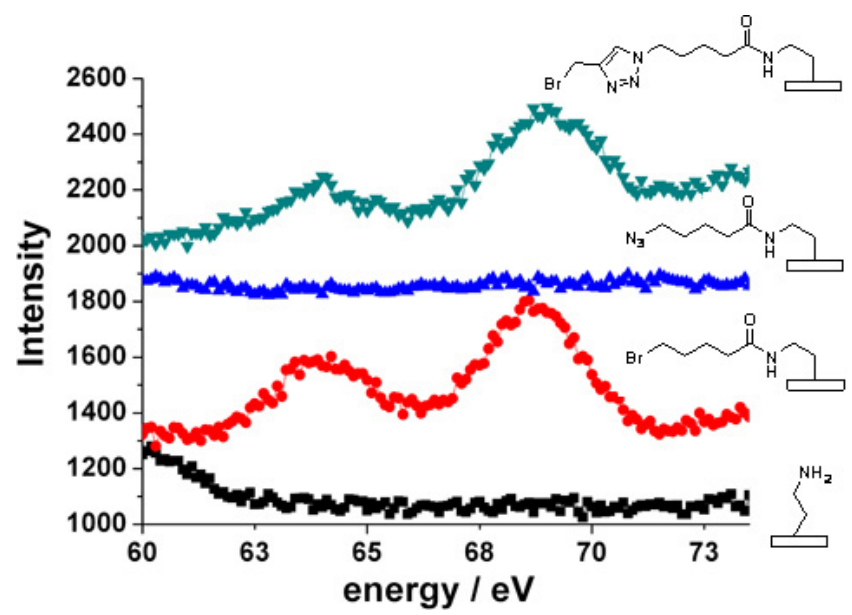

Fig. 1. Characterization of surface using XPS: Bottom to top: $\mathrm{Br} 3 \mathrm{~d}$ scan of aminated surface; after coupling with bromo acid; after azidation; after "clicking" with propargyl bromide. surface modification proceeds as planned by monitoring the appearance and dissaparance, then reappearance of the $\mathrm{Br} 3 \mathrm{~d}$ peaks at $63-70 \mathrm{eV}$. After coupling with the 5-bromo-pentanoic acid, the XPS $\mathrm{Br} 3 \mathrm{~d}$ peaks appear. Following that, the azidation step removes the bromide and replaces it with an azide group. Hence, we observe disappearance of the peaks. By choosing propargyl bromide as our alkyne substrate, we subsequently observe the appearance of the Br 3d peak again after the click chemistry step.

\subsection{Synthesis of propargyl 1-pyrene-butyrate}

1-pyrene-butyric acid (207 mg, $0.72 \mathrm{mmol}, 1.0$ equiv) was dissolved in chloroform $(24 \mathrm{~mL})$ in a round bottomed flask under nitrogen and cooled to $0^{\circ} \mathrm{C}$. Oxalyl chloride $(75 \mu \mathrm{L}, 0.86 \mathrm{mmol}, 1.2$ equiv) was then added slowly into the flask. Two drops of dimethyl formamide (DMF) were added as a catalyst for the reaction. The reaction mixture was stirred at room temperature for $20 \mathrm{~h}$ with constant stirring. The solvent and any excess oxalyl chloride was removed by rotary evaporation to obtain the acid chloride. The acid chloride was directly used in the next step without further purification.

The acid chloride (1.0 equiv) was dissolved in chloroform $(4 \mathrm{~mL})$ and added slowly to a solution of propargyl alcohol (142 $\mu \mathrm{L}, 1.08 \mathrm{mmol}, 1.5$ equiv) dissolved in chloroform $(24 \mathrm{~mL})$ and under nitrogen. The mixture was then stirred for $3 \mathrm{~h}$. The chloroform and excess propargyl alcohol were removed in vacuo The crude product was purified by flash chromatography (elution: hexane/DCM $=1 / 1$ to $\mathrm{DCM} /$ $\mathrm{MeOH}=95 / 5)$ to yield $118 \mathrm{mg}(50 \%)$ of the alkyne functionalized pyrene ester along with $99 \mathrm{mg}$ (48\%) of unreacted 1-pyrene-butyric acid. $\mathrm{R}_{f}$ (hexane/ $\mathrm{DCM}=1 / 1): 0.45 ;{ }^{1} \mathrm{H}-\mathrm{NMR}\left(400 \mathrm{MHz}, \mathrm{CDCl}_{3}\right) \delta$ $8.31-7.85\left(m, 9 \mathrm{H}, \mathrm{H}_{\text {arom }}\right), 4.71(d, J=2.5 \mathrm{~Hz}, 2 \mathrm{H}$, $\left.\mathrm{CH}_{2}\right), 3.43-3.39\left(m, 2 \mathrm{H}, \mathrm{CH}_{2}\right), 2.54-2.48(m, 3 \mathrm{H}$, $\mathrm{CH}_{2}$ and $\left.\mathrm{C} \equiv \mathrm{C}-\mathrm{H}\right), 2.26-2.17\left(m, 2 \mathrm{H}, \mathrm{CH}_{2}\right) ;{ }^{13} \mathrm{C}-$ NMR $\left(400 \mathrm{MHz}, \mathrm{CDCl}_{3}\right) \delta 172.7$ (COOR), 135.6 (C4), $131.6(\mathrm{C} 4), 131.1(\mathrm{C} 4), 130.2(\mathrm{C} 4), 128.9(\mathrm{C} 4)$, 127.6 (C3), 127.6 (C3), 127.5 (C3), 126.9 (C3), 126.0 (C3), 125.3 (C4), 125.1 (C4), 125.1 (C3), 125.0 (C3), 124.9 (C3), 123.4 (C3), 75.0 (R-C $\equiv \mathrm{C}), 52.0$ (C2), 51.7 (C $\equiv \mathrm{C}-\mathrm{H}), 33.7$ (C2), 32.8 (C2), 26.8 (C2); IR $(\mathrm{DCM}) \nu\left(\mathrm{cm}^{-1}\right) 3437,3304,3054,2987,2688,2306$, 1740, 1605, 1422, 1265, 1160, 1027, 896, 848, 740; ESI-MS: $\mathrm{m} / \mathrm{z}[\mathrm{M}+1]^{+}=327.4$. 


\subsection{Pyrene adsorption onto azido-quartz surfaces via click chemistry}

Following the demonstrated success of the click chemistry onto a quartz surface, we proceeded to attach pyrene onto the surface. 1-Pyrenebutyric acid was coupled with propargyl alcohol to yield the propargyl 1-pyrene butyrate, which was then used to "click" with the azide functionality on the quartz surface (Scheme 2).

The propargyl 1-pyrene-butyrate and $\mathrm{CuBr}$ were dissolved in DMF and the slides were incubated in the solution overnight with gentle shaking. The slides were then washed with water and acetone

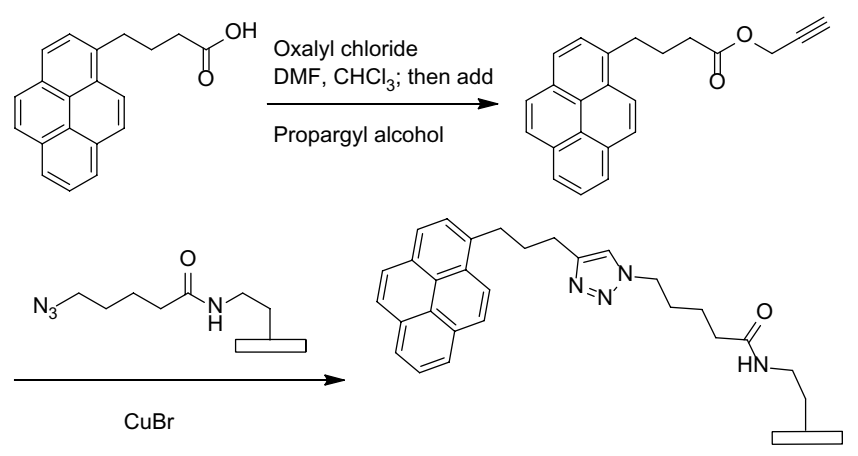

Scheme 2. Pyrene absorption onto quartz surface.
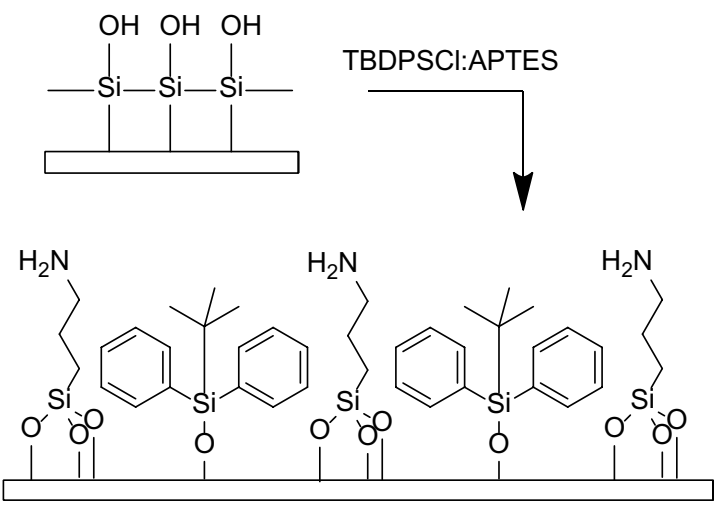

Scheme 3. Silyl spatial distribution of amine-attachment sites.

thoroughly. To dilute the distribution of amine on the quartz surface proportionally, tert-butyldiphenylchlorosilane (TBDPSCl) spacers at different mole ratios were introduced to take up some of the active space on the quartz slides. Practically, different molar ratios of TBDPSCl and APTES in the same solution were prepared and clean quartz slides were incubated in this media to produce spaced-out APTES (Scheme 3). These "diluted" slides could then be reacted as shown previously in Scheme 2 to provide the contact angles and ratios as given in Table 1 and 2, respectively.

Table 1. Contact angles determined after each reaction step.

\begin{tabular}{lc}
\hline Substrate & Contact angle (degree) \\
\hline Clean Quartz Slides & $3 \pm 2$ \\
Fully Aminated Quartz Slides & $72 \pm 2$ \\
Quartz Slides with Bromo Acid Linker & $80 \pm 3$ \\
Quartz Slides with Azidified Surface & $63 \pm 2$ \\
Quartz Slides after "Clicking" with Propagyl Bromide & $87 \pm 3$ \\
Quartz Slides after "Clicking" with Methyl Propiolate & $85 \pm 4$ \\
Quartz Slides after "Clicking" with Pyrene & $91 \pm 3$ \\
Quartz Slides Treated with 1:2 TBDPS-Cl:APTES & $68 \pm 4$ \\
Coupled to Bromo Acid Linker & $82 \pm 4$ \\
After Azidation & $71 \pm 4$ \\
After "Clicking" with Pyrene & $85 \pm 3$ \\
Quartz Slides Treated with 1:1 TBDPS-Cl:APTES & $71 \pm 2$ \\
Coupled to Bromo Acid Linker & $84 \pm 3$ \\
After Azidation & $73 \pm 4$ \\
After "Clicking" with Pyrene & $86 \pm 3$ \\
Quartz Slides Treated with 5:1 TBDPS-Cl:APTES & $72 \pm 3$ \\
Coupled to Bromo Acid Linker & $82 \pm 4$ \\
After Azidation & $71 \pm 3$ \\
After "Clicking" with Pyrene & $75 \pm 3$ \\
Quartz Slides Treated with 7:1 TBDPS-Cl:APTES & $74 \pm 3$ \\
Coupled to Bromo Acid Linker & $84 \pm 2$ \\
After Azidation & $72 \pm 4$ \\
After "Clicking" with Pyrene & $80 \pm 2$ \\
\hline
\end{tabular}


Table 1. (Continued)

\begin{tabular}{lc}
\hline Substrate & Contact angle (degree) \\
\hline Clean Quartz Slides & $3 \pm 2$ \\
Fully Aminated Quartz Slides & $72 \pm 2$ \\
Quartz Slides with Bromo Acid Linker & $80 \pm 3$ \\
Quartz Slides with Azidified Surface & $63 \pm 2$ \\
Quartz Slides after "Clicking" with Propagyl Bromide & $87 \pm 3$ \\
Quartz Slides after "Clicking" with Methyl Propiolate & $85 \pm 4$ \\
Quartz Slides after "Clicking" with Pyrene & $91 \pm 3$ \\
Quartz Slides Treated with 1:2 TBDPS-Cl:APTES & $68 \pm 4$ \\
Coupled to Bromo Acid Linker & $82 \pm 4$ \\
After Azidation & $71 \pm 4$ \\
After "Clicking" with Pyrene & $85 \pm 3$ \\
Quartz Slides Treated with 1:1 TBDPS-Cl:APTES & $71 \pm 2$ \\
Coupled to Bromo Acid Linker & $84 \pm 3$ \\
After Azidation & $73 \pm 4$ \\
After "Clicking" with Pyrene & $86 \pm 3$ \\
Quartz Slides Treated with 5:1 TBDPS-Cl:APTES & $72 \pm 3$ \\
Coupled to Bromo Acid Linker & $82 \pm 4$ \\
After Azidation & $71 \pm 3$ \\
After "Clicking" with Pyrene & $75 \pm 3$ \\
Quartz Slides Treated with 7:1 TBDPS-Cl:APTES & $74 \pm 3$ \\
Coupled to Bromo Acid Linker & $84 \pm 2$ \\
After Azidation & $72 \pm 4$ \\
After "Clicking" with Pyrene & $80 \pm 2$ \\
\hline
\end{tabular}

Table 2. Media used to aminate quartz slides with varying coverages.

\begin{tabular}{ccc}
\hline $\begin{array}{c}\text { Concentration of } \\
\text { TBDPS-Cl v/v /\% }\end{array}$ & $\begin{array}{c}\text { Concentration of } \\
\text { APTES v/v / } \%\end{array}$ & $\begin{array}{c}\text { Molar ratio of } \\
\text { TBDPS-Cl:APTES }\end{array}$ \\
\hline 0.85 & 1.52 & $1: 2$ \\
0.85 & 0.76 & $1: 1$ \\
0.85 & 0.15 & $5: 1$ \\
0.85 & 0.11 & $7: 1$ \\
\hline
\end{tabular}

\subsection{Spectroscopic analysis of pyrene-modified quartz surface}

Absorption spectra of the quartz slides were measured using a Shimadzu UV-2450 Spectrophotometer from 200-800 $\mathrm{nm}$. The emission from the quartz slides were measured using a Shimadzu RF-5301PC fluorimeter. The sample was excited at a wavelength of $329 \mathrm{~nm}$ and analyzed from $330 \mathrm{~nm}$ to $700 \mathrm{~nm}$. A UV-35 low pass filter was used for the excitation slit and a U-340 high pass filter was used for the emission slit. Excitation spectra were measured in the same fluorimeter.

\section{Results and Discussion}

Our first task was to determine the coverage of pyrene on the fully aminated quartz surface (Fig. 2). This was estimated using UV-Vis spectroscopy. By measuring the absorbance of the pyrene and using Beer-Lambert's law, we were able to estimate the coverage of the fully aminated quartz surface. Using an extinction coefficient of $54,000 \mathrm{~L} \mathrm{~mol}^{-1} \mathrm{~cm}^{-1},{ }^{24}$ an absorbance of 0.01 by pyrene at $349 \mathrm{~nm}$ would thus correspond to coverage of approximately 1 molecule $/ \mathrm{nm}^{2}$. The fully aminated quartz slide had an absorbance of approximately 0.017 , which corresponds to a coverage of about 0.85 molecule/ $\mathrm{nm}^{2}$. The value has to be divided by 2 as both sides of the quartz slide were aminated and the absorbance thus corresponds to two monolayers. The surface coverage could be determined for a range of pyrene: APTES ratios as shown in Fig. 2. Note that the extinction coefficient used here may be altered slightly by the fact that the pyrene is immobilized onto the quartz (vide infra).

The absorption spectra of the surfaces can be compared with the absorption spectra of pyrene and 

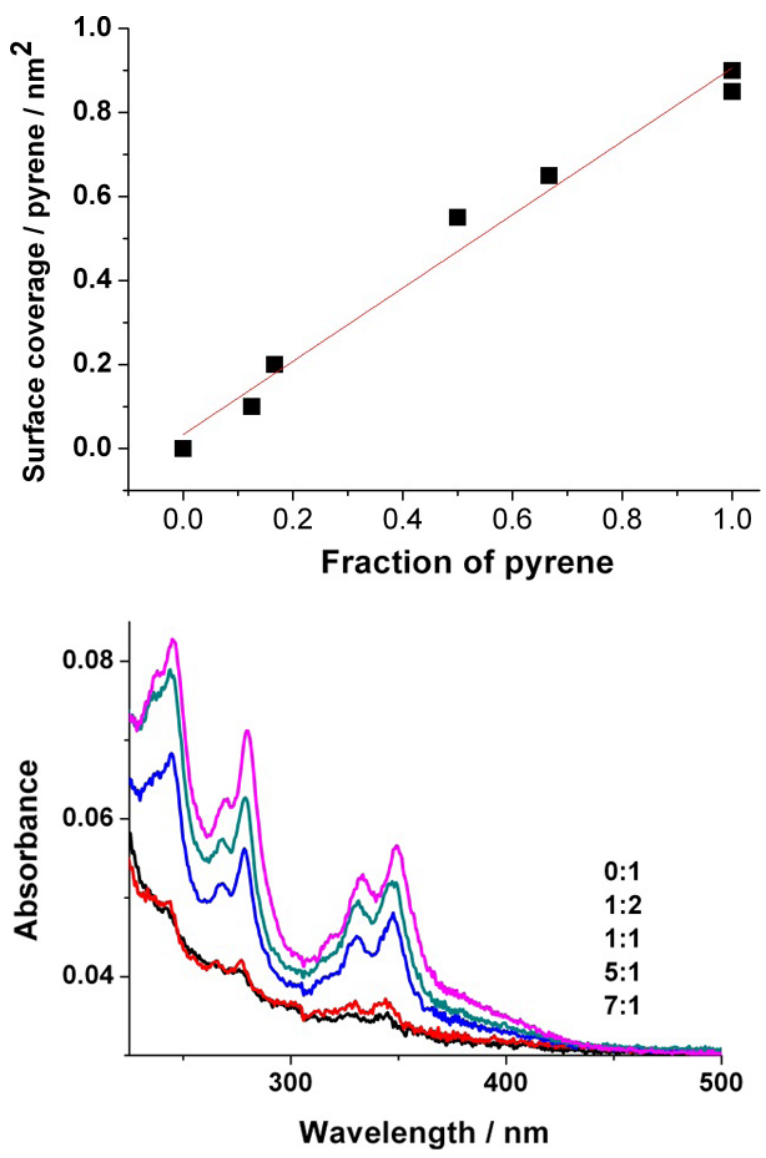

Fig. 2. Plot of pyrene-coverage against molar ratio of TBDS$\mathrm{Cl}$ :APTES (pyrene) used (top) and the spectra from which these points were derived (bottom).

the pyrene propargyl ester in ethanol solution as shown in Fig. 3.

From Fig. 3 we can see that the pyrene spectrum of the pyrene became significantly red-shifted on quartz compared to solution. Such red-shifts in the pyrene spectrum have been previously observed on surfaces and in cellulose polymers and is accounted for by loose ground state association. ${ }^{25}$ Probably the surface coverage of pyrene that is achieved after click-chemistry means that they are in much closer proximity than they would usually exist in solution.

The coverage dependence of the red-shift may then be due to the loss of interactions with pyrene nearest the neighbors, as they get replaced by with TBDSCl. Another salient point is the long wavelength tail in the absorption spectrum of the pyrene seen in Fig. 3. The tail becomes more prominent at higher surface coverage of pyrene. Therefore this longer tail in the absorption and excitation spectra can be ascribed to forced $\pi$-cloud interactions between pyrene molecules even in the ground-state
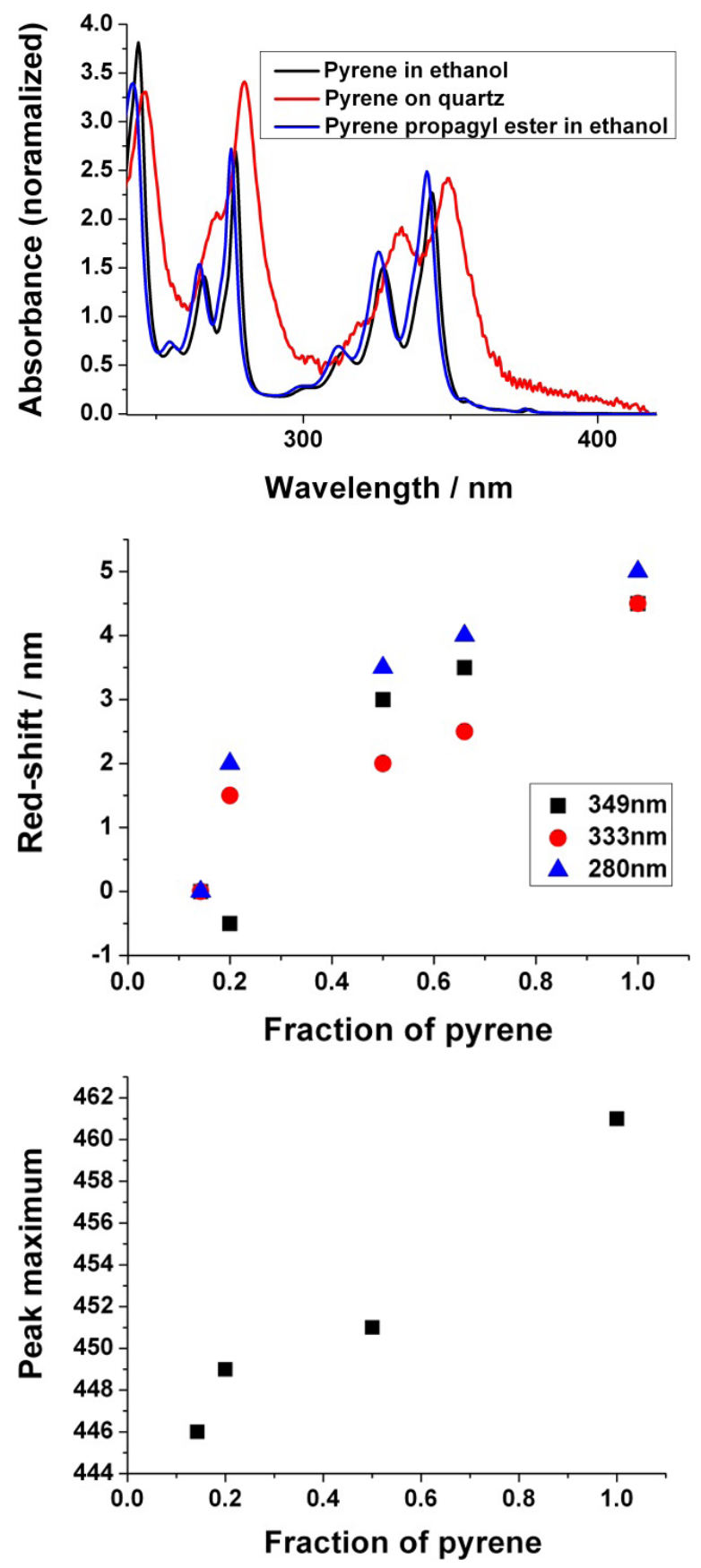

Fig. 3. Spectral shifts observed for pyrene on quartz at different surface coverage. Top: Comparison with solution is also shown. Middle: Spectral shifts in absorption are shown, Bottom: Spectral shifts of the excimer broad emission is shown.

leading to direct intermolecular charge transfer absorption. In the current case, this may be observed due to the highly dense packing of the pyrene molecules.

Next, the fluorescence of the pyrene on the quartz slides was studied. The excitation spectra obtained (monitoring in the excimer region) is a more or less 

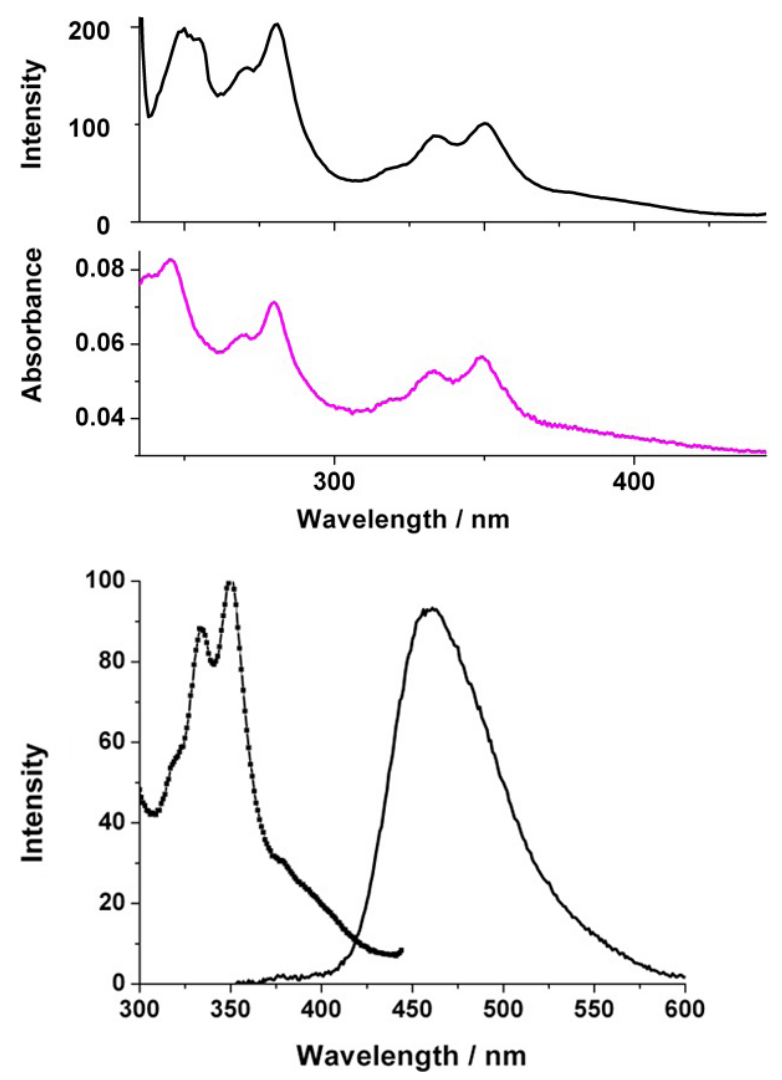

Fig. 4. Absorption, emission and excitation spectra of pyrene on a fully covered quartz surface.

perfect superposition of the absorption spectrum, including the red-shift and the tail into the longer wavelength region as shown in Fig. 4. This confirms that the broad emission, ascribed here as a CT band, does indeed come from excitation of the pyrene, and also not from some surface contaminant or absorption from the substrate. The emission from the excimer overlaps well with the tail in the absorption (and excitation) spectra. However, this absorption tail extends further into the red than the highest energy vibrational band in the monomer emission. This does indicate that the tail is due to some interaction between ground-state monomers and that excitation of this tail should not yield emission from the monomers as the energy of light in the absorption tail is lower than that from the highest energy vibrational band of the monomer.

The emission spectrum of the fully aminated slides show strong excimer fluorescence at $460 \mathrm{~nm}$ and almost no monomer fluorescence (Fig. 5). These results show not only the presence of pyrene immobilized on the fully aminated quartz surface but also that the coverage is so high that pyrene
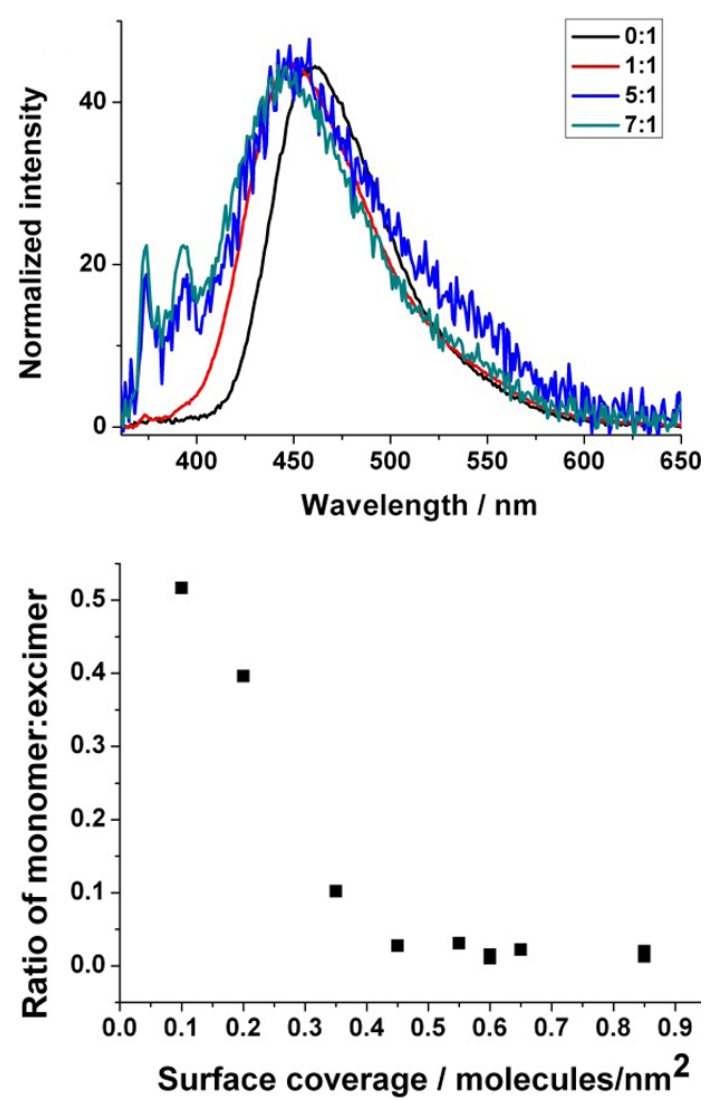

Fig. 5. Plot of monomer/excimer ratio against pyrene coverage (as calculated from signal integrals in emission spectra).

molecules form excimers exclusively. This again suggests that there is very close packing of pyrene on the surface as the molecules are essentially tethered and cannot diffuse to form excimer other than wagging and having rotational motions. As the amount of TBDSCl spacer is increased, some monomer emission is observed. The average distance of two pyrene molecules in the excimer is expected to be $\sim 3.53 \AA$ (Ref. 1 ) as it is in the crystal state. The appearance of the monomer is therefore expected when the average distance between the pyrene molecules becomes sufficient that some of them cannot touch their nearest pyrene neighbors. This will happen when the chain length is not long enough for the pyrene molecules to interact effectively with one another.

By plotting the monomer/excimer ratio against the coverage, we are able to approximate the average coverage where monomers start to form (Fig. 5). At an average coverage of 0.45 molecule $\mathrm{nm}^{-2}$, the pyrene begins to form monomers.

The formation of pyrene excimer requires the two pyrene molecules to stack face to face. At a high 

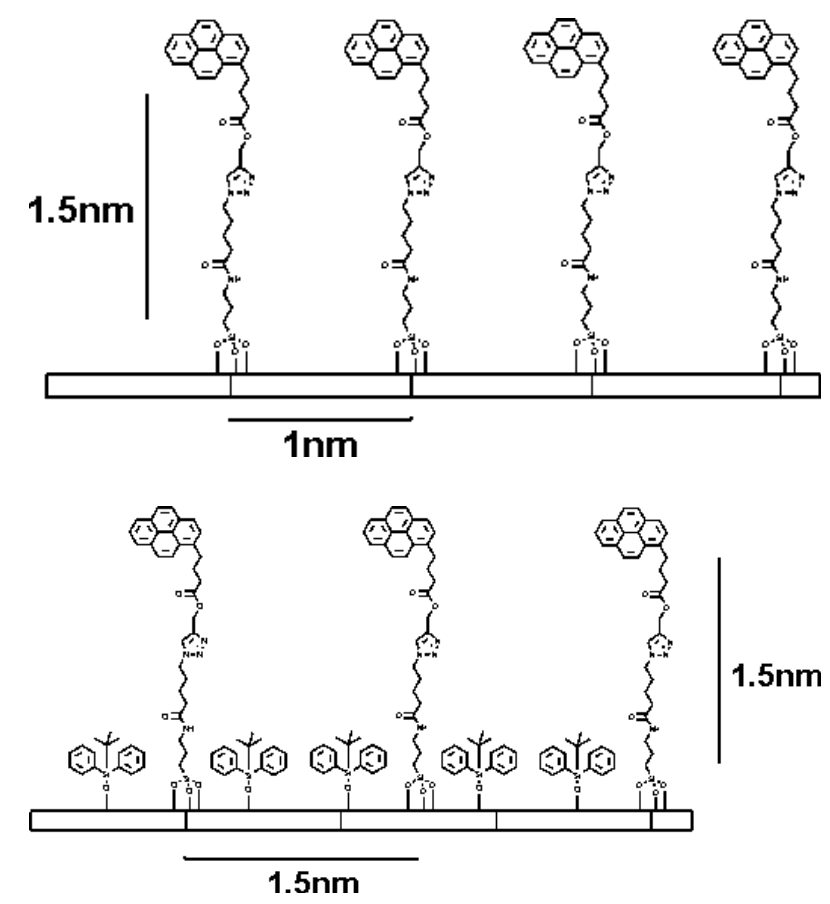

Fig. 6. Estimated scale drawing of monolayer at different coverage: (top) monolayer at Coverage of 0.8 molecule $\mathrm{nm}^{-2}$; (bottom) monolayer at coverage of 0.45 molecule $\mathrm{nm}^{-2}$.

coverage, this is not expected to be a problem as the pyrene molecules are very close together. The average distance between two tether chains at the anchor point to the surface is about $1 \mathrm{~nm}$ at 0.8 molecule $\mathrm{nm}^{-2}$. The flexible portion of the chain is approximately $1.5 \mathrm{~nm}$ long. Hence at an average separation of $1 \mathrm{~nm}$, it is still relatively easy for the pyrene to stack with one another to form an excimer.

However, at an average coverage of 0.45 molecule $\mathrm{nm}^{-2}$, the average separation of the chains at the anchor is approximately $1.5 \mathrm{~nm}$ (Fig. 6). At this distance, the chains begin to have to strain for the pyrene molecules to effectively stack with one another. Thus we begin to see monomer fluorescence at this coverage. As the coverage is further reduced, it becomes harder for the pyrene to stack effectively; hence we start to see a much larger amount of monomer fluorescence. Of course we must remember that this is the average separation and that there will be a distribution of intermolecular separations at the surface. However, it is an interesting rule of thumb that when the chain length is equal to the average separation some of the molecules begin to find difficulty in interacting.

One may reasonably question whether the amine groups on the linker chains themselves can form an intramolecular exciplex with the pyrene. It is well known that such an intramolecular exciplex can form in solution, therefore, it cannot be ruled out that at least some of the broad emission seen in the present study results from an exciplex. In answer to this, due to the TBDSCl dependence of the monomer to excimer ratio, we can state that excimers must be a factor, however, some exciplex cannot be ruled out. Other groups have reported exclusively monomer emission for pyrene immobilized on surfaces where the surface coverage is just under two orders of magnitude lower than in the current case, however, in these studies the amine portion of the linker was just 2-bonds from the pyrene, so it would be sterically difficult for the exciplex to form. ${ }^{5}$ However, in the same work, when the pyrene was diluted with a long chain amino acid spacer, that was abundant enough to affect the monomer vibrational bands by hanging local polarity, there was still no evidence for exciplex. ${ }^{5}$ For this we reason that the broad emission is at least predominantly excimer, as suggested by the TBDSCl-content dependence of the ratio of monomer to excimer. Computational studies also do not suggest the formation of exciplex in close packed chains terminated with pyrene for coverages of $\sim 1.1$ molecules $/ \mathrm{nm}^{2}$. In that work, the pyrenes were instead found to form loose associations with distances $\sim 3.5 \AA .^{26}$ This finding is also consistent with the observation of CT-absorption in the spectra obtained in the current work.

Last, we note that the broad excimer emission red shifts as the coverage of APTES, and hence the pyrene, is increased (Fig. 3). This may be due local polarity or other environmental dependencies of the excimer. It may also indicate a small contribution of exciplex to the overall broad spectrum.

\section{Conclusion}

We have successfully used alkyne-azide click chemistry to form high density packing of pyrene at a quartz surface. The packing density is so great that when no spacers are used to dilute the pyrene, ground state association occurs leading to spectral shifts in the absorption spectra. Furthermore, a CT absorption develops at a longer wavelength. This suggests that direct formation of excimer from this intermolecular CT from pyrene to pyrene is possible during the primary photochemical step (the absorption itself). The excimer emission dominates at 
all coverage studies here, however, at lower coverages the monomer emission can also be observed when the average distance between the anchor points is similar to the pyrene's linker chain length. This may make a useful rule of thumb when judging at what coverages molecules begin to struggle to interact.

\section{Acknowledgments}

The authors are grateful to A-STAR - JCO (JH) and A-STAR-JST (MJL, JH, SG, SHY) for funding this work.

\section{References}

1. A. Gilbert and J. Baggott, Essentials of Molecular Photochemistry (Blackwell Scientific Publications, UK, 1991).

2. K. Yoshihara, T. Kasuya, A. Inoue and S. Nakakura, Chem. Phys. Lett. 9(5), 469 (1971).

3. H. Fujiwara, K. Sasaki and H. Masuhara, J. Appl. Phys. 85(4), 2052 (1999).

4. S. Funakura, K. Nakatani, H. Misawa, N. Kitamura and H. Masuhara, J. Phys. Chem. 99, 15192 (1995).

5. M. Nakamura, N. Saito and K. Takayama, Chem. Lett. 36, 602 (2007).

6. M. Mazur and G. J. Blanchard, J. Phys. Chem. B 109, 4076 (2005).

7. L. N. Gao, Y. Fang, F. T. Lü and L. P. Ding, Sci. China Ser. B 47, 240 (2004).

8. A. D'Aléo, E. Cecchetto, L. De Cola and R. M. Williams, Sensors 9(5), 3604 (2009).

9. L. N. Gao, Y. Fang, X. P. Wen, Y. G. Li and D. D. Hu, J. Phys. Chem. B 108, 1207 (2004).

10. L. N. Gao, Y. Fang, F. T. Lü, M. W. Cao and L. P. Ding, Appl. Surf. Sci. 252, 3884 (2006).
11. F. T. Lü, Y. Fang, L. N. Gao, L. P. Ding and L. L. Jiang, J. Photochem. Photobiol. A 175, 207 (2005).

12. H. C. Kolb, M. G. Finn and K. B. Sharpless, Angew. Chem. Int. Ed. 40, 2004 (2001).

13. C. W. Tornoe, C. Christensen and M. Meldal, J. Org. Chem. 67, 3057 (2002).

14. V. V. Rostovtsev, L. G. Green, V. V. Fokin and K. B. Sharpless, Angew. Chem. Int. Ed. 41, 2596 (2002).

15. R. Srinivasan, J. Li, S. L. Ng, K. A. Kalesh and S. Q. Yao, Nat. Prot. 2, 1 (2007).

16. J. Wang, M. Uttamchandani, J. Li, M. Hu and S. Q. Yao, Org. Lett. 8, 3821 (2006).

17. M. Malkoch, K. Schleicher, E. Drockenmuller, C. J. Hawker, T. P. Russell, P. Wu and V. V. Fokin, Macromolecules 38, 3663 (2005).

18. G. K. Such, J. F. Quinn, A. Quinn, E. Tjipto and F. Caruso, J. Am. Chem. Soc. 128, 9318 (2006).

19. J. P. Collman, N. K. Devaraj and C. E. D. Chidsey, Langmuir 20, 1051 (2004).

20. T. Lummerstorfer and H. Hoffman, J. Phy. Chem. B 108, 3963 (2004).

21. D. I. Rozkiewicz, D. Janczewski, W. Verboom, B. Jan Ravoo and D. N. Reinhoudt, Angew. Chem. Int. Ed. 45, 5292 (2006).

22. R. Srinivasan, M. Uttamchandani and S. Q. Yao, Org. Lett. 8, 713 (2006).

23. B. A. Laurent and S. M. Grayson, J. Am. Chem. Soc. 128, 4238 (2006).

24. I. B. Berlman, Handbook of Fluorescence Spectra of Aromatic Molecules (Academic Press, New York, 1971).

25. A. S. Oliveira, L. F. V. Ferreira, J. P. Da Silva and J. C. Moreira, Int. J. Photoenergy 6, 205 (2004).

26. J. Liu, Y. Fang and C. L. Chen, Langmuir 24, 1853 (2008). 